\title{
Estimation of Skin Factor for Wells in Tight Gas Reservoirs
}

D. Sun* (Baker Hughes Incorporated), D. Georgi (Baker Hughes Inc.), G. Michel (University of Oklahama), R. Pour (Unviersity of Austin), J. Wu (Baker Hughes Inc.), B. Li (Baker Hughes Inc.), H. Grimmer (Baker Hughes Inc.), A. Jacques (Total) \& J. Lassus-Dessus (Total)

\section{SUMMARY}

Skin factor is a common measure of formation damage and is an important input parameter for reservoir simulation of tight gas (TG) reservoirs. We explored, with reservoir simulation, production from TG reservoirs without hydraulic fracturing and incorporated a new model for drilling induced skin. We undertook an extensive sensitivity study to estimate the TG reservoir's skin factor. In the study, we developed a three-concentric-layered damaged zone parameterization. We explored different borehole sizes and found that the skin factor decreases when formation permeability anisotropy increases, assuming the same horizontal invasion depth and the same damaged permeability ratio.

A near-wellbore reservoir simulation model was selected for our application. In the study, we analyzed the effect of the filter cake during over-balanced drilling operation with water-based mud and its effect on the gas production. The distributions of water saturation and effective gas permeability in the example have been calculated during invasion and after production. The total skin factor is calculated based on averaging method. We found the filter cake has a minor effect on the depth of invasion due to the low effective permeability of the formation. Core testing of mud invasion/production are needed for verification. 
Formation damage due to drilling in a tight gas (TG) reservoir is not the only reason but is a key reason for the widespread use of hydraulic fracturing in the production of TG reservoirs. Sometimes, for example near water zones, hydraulic fracturing cannot be controlled well enough to be used to overcome formation damage and produce TG reservoirs. Skin factor is a common measure of formation damage and is an important input parameter for reservoir simulation of TG reservoirs. We explored, with reservoir simulation, production from TG reservoirs without hydraulic fracturing and incorporated a new model for drilling induced skin.

For our studies we reviewed pertinent formation damage mechanisms for TG reservoirs, including phase trapping/water blocking; fines migration/solid invasion; and clay swelling. We undertook an extensive sensitivity study to estimate the TG reservoir's skin factor. In our simulations, we also considered the effects of permeability anisotropy $(\mathrm{Kh} / \mathrm{Kv}=1,10$ and100) in the TG formation. For the sensitivity study, we developed a three-concentric-layered damaged zone parameterization. Each of the three layers could be assigned different thicknesses and return permeabilities. For our study, we explored different borehole sizes and found that the skin factor of a slim borehole with a smaller diameter is more sensitive to invasion thickness than that of a slim borehole with a larger diameter. The skin factor decreases when formation permeability anisotropy increases, assuming the same horizontal invasion depth and the same damaged permeability ratio.

Details on modeling TG formation damage and setting up of the simulation are elaborated. A nearwellbore model was selected for our application. In the study, we analyzed the effect of the filter cake during over-balanced drilling operation with water-based mud and its effect on the gas production. The distributions of water saturation and effective gas permeability in the example have been calculated during invasion and after production. The skin factors at different angles are calculated based on radial effective gas permeability in the damaged zone. The total skin factor is calculated based on averaging method. We found the filter cake has a minor effect on the depth of invasion due to the low effective permeability of the formation. Water blocking, assuming that the initial water saturation is equal to the irreducible water saturation, does not affect much on the production rate curve comparing with no formation damage. Core testing and more numerical simulations of mud invasion/production are needed for different tight gas formations. 